\title{
The importance of being random: statistical principles of iris recognition
}

\author{
John Daugman* \\ The Computer Laboratory, University of Cambridge, Cambridge CB3 OFD, UK
}

Received 21 December 2001

\begin{abstract}
The statistical variability that is the basis of iris recognition is analysed in this paper using new large databases. The principle underlying the recognition algorithm is the failure of a test of statistical independence on iris phase structure encoded by multi-scale quadrature wavelets. Combinatorial complexity of this phase information across different persons spans about 249 degrees-of-freedom and generates a discrimination entropy of about $3.2 \mathrm{bits} / \mathrm{mm}^{2}$ over the iris, enabling real-time identification decisions with great enough accuracy to support exhaustive searches through very large databases. This paper presents the results of 9.1 million comparisons among several thousand eye images acquired in trials in Britain, the USA, Japan and Korea. (C) 2002 Pattern Recognition Society. Published by Elsevier Science Ltd. All rights reserved.
\end{abstract}

Keywords: Statistical variability; Epigenesis; Wavelets; Texture; Iris recognition; Decision theory

\section{Introduction}

Randomness plays a crucial role in many sciences, and increasingly also for technologies. In biology, random variation by mutation is the engine of evolution; in physics, random state variables are key to quantum mechanics and to thermodynamics; and in the information sciences, random sequences are central to theories of cryptography, data compressibility, and algorithmic complexity. Many methods exist for measuring and describing the randomness of variables or patterns. Perhaps the most interesting of these are the proposals by the Russian mathematician Kolmogorov [1] that the complexity of a random sequence is equal to the length of the shortest program that can generate it, and that a pattern is defined to be algorithmically random if it is its own shortest possible description.

Biometric identification systems all rely upon forms of random variation among persons. The more complex the randomness the better, because more dimensions

* Tel.: +44-1223-334501; fax: +44-1223-334678.

E-mail address: john.daugman@cl.cam.ac.uk (J. Daugman). of independent variation produce signatures having greater uniqueness. But while seeking to maximize the between-person variability, biometric templates must also have minimal within-person variability across time and changing conditions of capture. In the case of face recognition, for example, difficulties arise from the fact that faces are changeable social organs displaying a variety of expressions, as well as being active 3D objects whose projected images vary with pose and viewing angle, illumination, accoutrements, and age. Against this within-person (same face) variability, between-person variability is limited because different faces possess the same canonical set of features, always in basically the same canonical geometry. As is easily illustrated, see Refs. [2,3], the variability among frontal images of any given face even just from illumination alone can be much larger than the variability among images of different faces captured with fixed expression; and it has been noted that for images taken at least $1 \mathrm{yr}$ apart, even the best face recognition algorithms have error rates from $43 \%$ [4] to $50 \%$ [5].

For all of these reasons, iris patterns become interesting as an alternative approach to reliable visual recognition of persons, when imaging can be done at distances of about a 


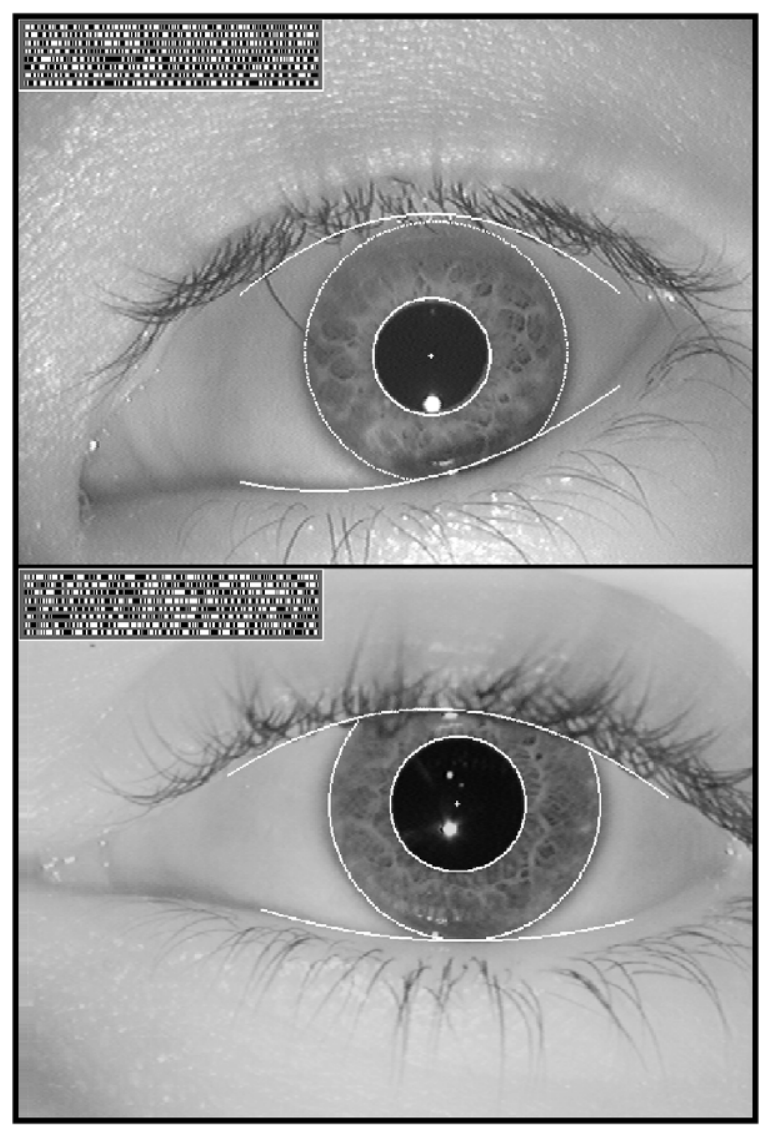

Fig. 1. Examples of iris patterns, imaged monochromatically with NIR illumination in the $700-900 \mathrm{~nm}$ band at distances of about $35 \mathrm{~cm}$. The outline overlays show results of the iris and pupil localization and eyelid detection steps. The bit streams are the results of demodulation with complex-valued 2D Gabor wavelets to encode iris patterns as a sequence of phasor quadrants.

meter or less, and especially when there is a need to search very large databases. Although small $(11 \mathrm{~mm})$ and sometimes problematic to image, the iris has the great mathematical advantage that its pattern variability among different persons is enormous. In addition, as an internal (yet externally visible) organ of the eye, the iris is well protected from the environment, and stable over time. As a planar object, its image is relatively insensitive to angle of illumination, and changes in viewing angle cause only reversible affine transformations; even the non-affine pattern distortion caused by pupillary dilation is readily reversible. Finally, the ease of localizing eyes in faces, and the distinctive annular shape of the iris, facilitate reliable and precise isolation of this feature and the creation of a size-invariant representation.

The iris begins to form in the third month of gestation [6] and the structures creating its pattern are largely complete by the eighth month, although pigment accretion can continue into the first postnatal years. Its complex pattern can contain many distinctive features such as arching ligaments, furrows, ridges, crypts, rings, corona, freckles, and a zigzag collarette, some of which may be seen in the two irises in Fig. 1. The striated trabecular meshwork of elastic pectinate ligament creates the predominant texture under visible light, whereas in the near-infrared (NIR) wavelengths used for unobtrusive imaging at distances of up to $1 \mathrm{~m}$, deeper and somewhat more slowly modulated stromal features dominate the iris pattern. In NIR wavelengths, even darkly pigmented irises reveal rich and complex features.

Methods for encoding and recognizing iris patterns were first described in 1993 by Daugman [7]. These algorithms, released as executables, have been the basis of all iris recognition systems so far deployed in public trials, including those by British Telecom, US Sandia Labs, UK National Physical Laboratory, The National Biometric Test Center of SJSU, EyeTicket, Siemens, Unisys, LG, IriScan, Iridian, Sensar, and Sarnoff. All of these organizations have reported a false match rate of zero in all of their tests, some of which involved millions of different iris pairings. High volume deployments have now begun at international airports Heathrow, Schiphol, Frankfurt, and Charlotte. This paper describes how the algorithms work and explains improvements over the author's original 1993 methods; presents new data on the statistical properties and singularity of iris patterns based on 9.1 million comparisons; and discusses future developments that are needed.

\section{Localizing and isolating an iris}

To capture the rich details of iris patterns, an imaging system should resolve a minimum of 50 pixels in iris radius. In the field trials to date, a resolved iris radius of 100-140 pixels has been more typical. Monochrome CCD cameras $(480 \times 640)$ have been used because NIR illumination in the $700-900 \mathrm{~nm}$ band was required for imaging to be invisible to humans. Some imaging platforms deployed a wide-angle camera for coarse localization of eyes in faces, to steer the optics of a narrow-angle pan/tilt camera that acquired higher resolution images of the eyes. But most of the imaging was done without active pan/tilt camera optics, instead exploiting visual feedback via a mirror or video image to enable cooperating Subjects to position their own eyes within the field of view of a single narrow-angle camera.

Focus assessment was performed in real-time (faster than video frame rate) by measuring the total high-frequency power in the 2D Fourier spectrum of each frame, and seeking to maximize this quantity either by moving an active lens or by providing audio feedback to Subjects to adjust their range appropriately. Images passing a minimum focus criterion were then analysed to find the iris, with precise localization of its boundaries using a coarse-to-fine strategy 
terminating in single-pixel precision estimates of the centre coordinates and radius of both the iris and the pupil. Although the results of the iris search greatly constrain the pupil search, concentricity of these boundaries cannot be assumed. Very often the pupil centre is nasal, and inferior, to the iris centre. Its radius can range from 0.1 to 0.8 of the iris radius. Thus, all three parameters defining the pupillary circle must be estimated separately from those of the iris. A very effective integrodifferential operator for determining these parameters is

$\max _{\left(r, x_{0}, y_{0}\right)}\left|G_{\sigma}(r) * \frac{\partial}{\partial r} \oint_{r, x_{0}, y_{0}} \frac{I(x, y)}{2 \pi r} \mathrm{~d} s\right|$,

where $I(x, y)$ is an image as in Fig. 1 containing an eye. The operator searches over the image domain $(x, y)$ for the maximum in the blurred partial derivative with respect to increasing radius $r$ of the normalized contour integral of $I(x, y)$ along a circular arc $\mathrm{d} s$ of radius $r$ and centre coordinates $\left(x_{0}, y_{0}\right)$. The symbol ${ }^{*}$ denotes convolution and $G_{\sigma}(r)$ is a smoothing function such as a Gaussian of scale $\sigma$. The complete operator behaves in effect as a circular edge detector, blurred at a scale set by $\sigma$, which searches iteratively for a maximum contour integral derivative with increasing radius at successively finer scales of analysis through the three parameter space of centre coordinates and radius $\left(x_{0}, y_{0}, r\right)$ defining a path of contour integration.

The operator in Eq. (1) serves to find both the pupillary boundary and the outer (limbus) boundary of the iris, although the initial search for the limbus should incorporate evidence of an interior pupil to improve its robustness since the limbic boundary itself usually has extremely soft contrast when long-wavelength NIR illumination is used. Once the coarse-to-fine iterative searches for both these boundaries have reached single pixel precision, then a similar approach to detecting curvilinear edges is used to localize both the upper and lower eyelid boundaries. The path of contour integration in Eq. (1) is changed from circular to arcuate, with spline parameters fitted by standard statistical estimation methods to describe optimally the available evidence for each eyelid boundary. The result of all these localization operations is the isolation of iris tissue from all other image regions, as illustrated in Fig. 1 by the graphical overlays on these two eyes.

\section{Iris feature encoding by 2D wavelet demodulation}

Each isolated iris pattern is then demodulated (see Ref. [8]) to extract its phase information using quadrature 2D Gabor wavelets (see Refs. [9-11]). This encoding process is illustrated in Fig. 2. It amounts to a patch-wise phase quantization of the iris pattern, by identifying in which quadrant of the complex plane each resultant phasor lies when a given area of the iris is projected onto complex-valued 2D
Gabor wavelets:

$$
\begin{aligned}
h_{\{R e, I m\}}= & \operatorname{sgn}_{\{R e, I m\}} \int_{\rho} \int_{\phi} I(\rho, \phi) \mathrm{e}^{-\mathrm{i} \omega\left(\theta_{0}-\phi\right)} \mathrm{e}^{-\left(r_{0}-\rho\right)^{2} / \alpha^{2}} \\
& \times \mathrm{e}^{-\left(\theta_{0}-\phi\right)^{2} / \beta^{2}} \rho \mathrm{d} \rho \mathrm{d} \phi,
\end{aligned}
$$

where $h_{\{R e, I m\}}$ can be regarded as a complex-valued bit whose real and imaginary parts are either 1 or 0 (sgn) depending on the sign of the $2 \mathrm{D}$ integral; $I(\rho, \phi)$ is the raw iris image in a dimensionless polar coordinate system that is size- and translation-invariant, and which also corrects for pupil dilation as explained in a later section; $\alpha$ and $\beta$ are the multi-scale $2 \mathrm{D}$ wavelet size parameters, spanning an 8 -fold range from 0.15 to $1.2 \mathrm{~mm}$ on the iris; $\omega$ is wavelet frequency, spanning three octaves in inverse proportion to $\beta$; and $\left(r_{0}, \theta_{0}\right)$ represent the polar coordinates of each region of iris for which the phasor coordinates $h_{\{R e, I m\}}$ are computed. Such a phase quadrant coding sequence is illustrated for two irises by the bit streams pictured in Fig. 1. A desirable feature of the phase code explained in Fig. 2 is that it is a cyclic or Gray code: in rotating between any adjacent phase quadrants, only a single bit changes, unlike a binary code in which two bits may change, making some errors arbitrarily more costly than others. Altogether 2048 such phase bits (256 bytes) are computed for each iris, but in a major improvement over the earlier Daugman [7] algorithms, now an equal number of masking bits are also computed to signify whether any iris region is obscured by eyelids, contains any eyelash occlusions, specular reflections, boundary artifacts of hard contact lenses, or poor signal-to-noise ratio and thus should be ignored in the demodulation code as artifact.

Only phase information is used for recognizing irises because amplitude information is not very discriminating, and it depends upon extraneous factors such as imaging contrast, illumination, and camera gain. The phase bit settings which code the sequence of projection quadrants as shown in Fig. 2 capture the information of wavelet zero-crossings, as is clear from the sign operator in Eq. (2). The extraction of phase has the further advantage that phase angles are assigned regardless of how poor the image contrast may be, as illustrated by the extremely out-of-focus image in Fig. 3. Its phase bit stream has statistical properties such as run lengths similar to those of the code for the properly focused eye images in Fig. 1. (Fig. 3 also illustrates the robustness of the iris- and pupil-finding operators, and the eyelid detection operators, despite poor focus.) The benefit which arises from the fact that phase bits are set also for a poorly focused image as shown here, even if based only on random CCD noise, is that different poorly focused irises never become confused with each other when their phase codes are compared. By contrast, images of different faces look increasingly alike when poorly resolved, and may be confused with each other by face recognition algorithms. 


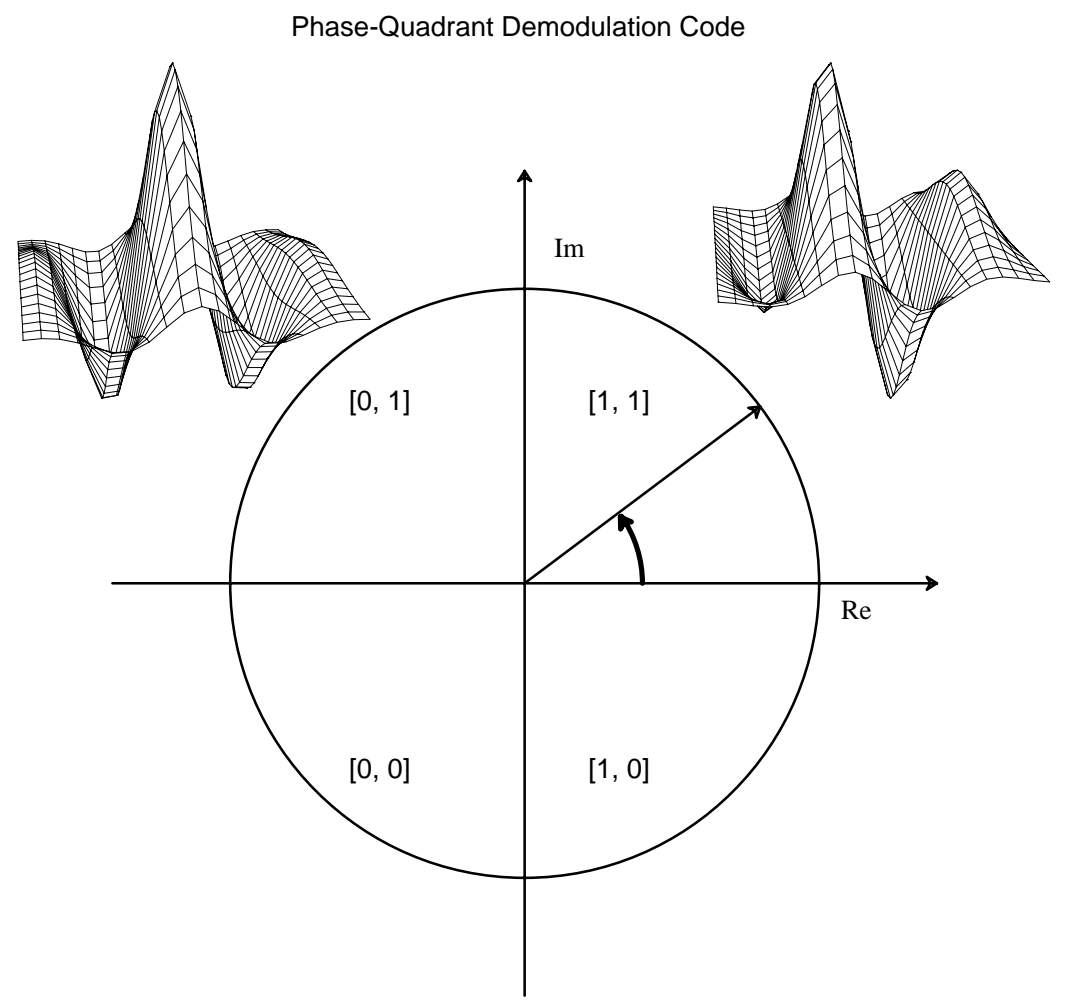

Fig. 2. The phase demodulation process used to encode iris patterns. Local regions of an iris are projected (Eq. (2)) onto quadrature 2D Gabor wavelets, generating complex-valued projection coefficients whose real and imaginary parts specify the coordinates of a phasor in the complex plane. The angle of each phasor is quantized to one of the four quadrants, setting two bits of phase information. This process is repeated all across the iris with many wavelet sizes, frequencies, and orientations, to extract 2048 bits.

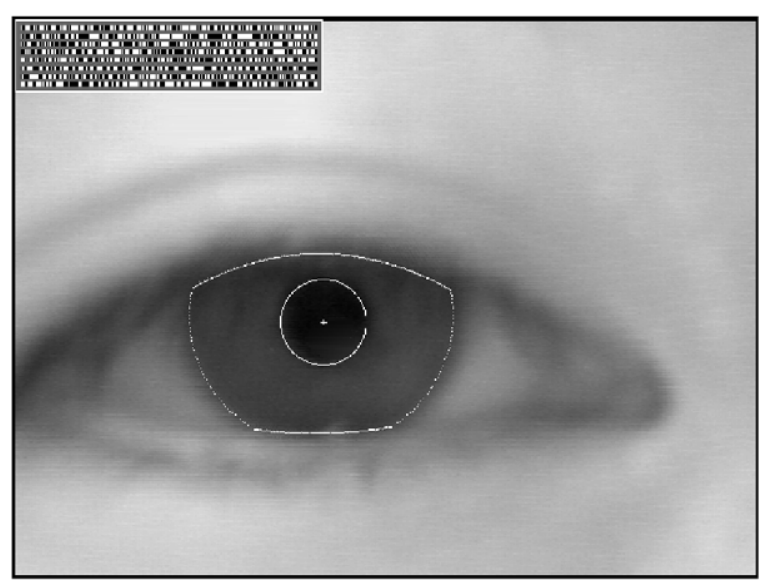

Fig. 3. Illustration that even for poorly focused eye images, the bits of a demodulation phase sequence are still set, primarily by random CCD noise. This prevents poorly focused eye images from resembling each other in the pattern matching stage, in the way that, e.g., poorly resolved face images look alike and can be confused with each other.

\section{The test of statistical independence: combinatorics of phase sequences}

The key to iris recognition is the failure of a test of statistical independence, which involves so many degrees-of-freedom that this test is virtually guaranteed to pass whenever the phase codes for two different eyes are compared, but it uniquely fails when any eye's phase code is compared with another version of itself.

The test of statistical independence is implemented by the simple Boolean exclusive-OR operator (XOR) applied to the 2048 bit phase vectors that encode any two iris patterns, masked (AND'ed) by both of their corresponding mask bit vectors to prevent non-iris artifacts from influencing iris comparisons. The XOR operator $\otimes$ detects disagreement between any corresponding pair of bits, while the AND operator $\cap$ ensures that the compared bits are both deemed to have been uncorrupted by eyelashes, eyelids, specular reflections, or other noise. The norms ( \|\| ) of the resultant bit vector and of the AND'ed mask vectors are then measured in order to compute a fractional Hamming distance (HD) as a measure of the dissimilarity between any two irises, whose 


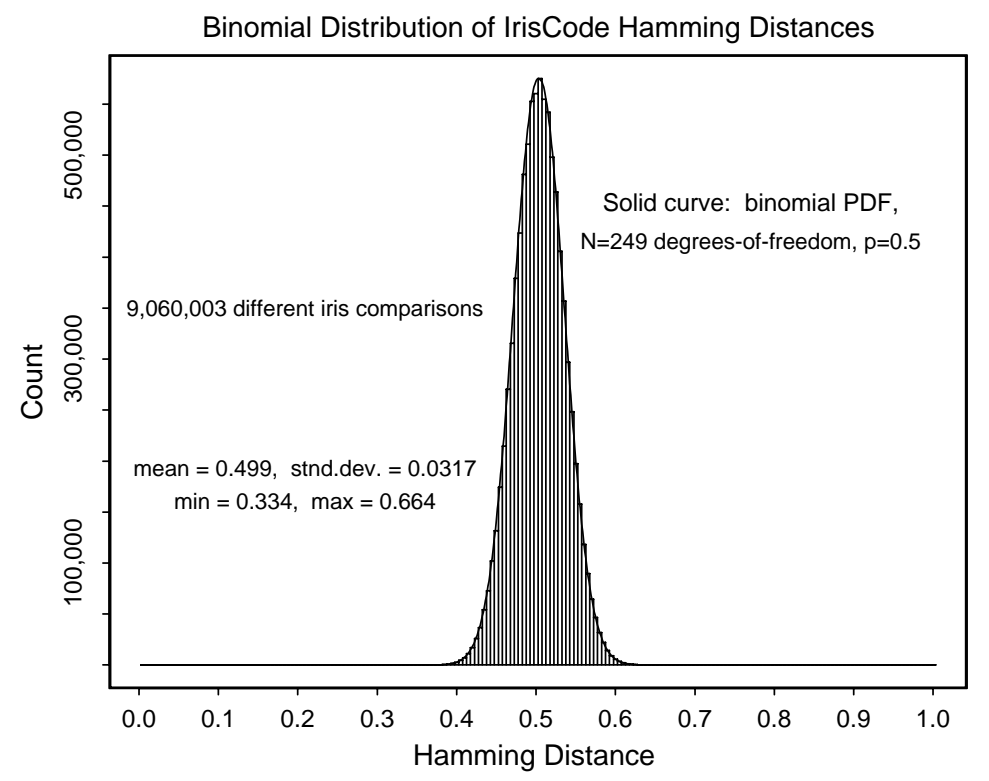

Fig. 4. Distribution of Hamming distances obtained from all 9.1 million possible comparisons between different pairs of irises in the database. The histogram forms a perfect binomial distribution with $p=0.5$ and $N=249$ degrees-of-freedom, as shown by the solid curve (Eq. (4)). The data implies that it is extremely improbable for two different irises to disagree in less than about a third of their phase information.

two phase code bit vectors are denoted $\{$ codeA, codeB $\}$ and whose mask bit vectors are denoted $\{$ maskA, maskB\}:

$H D=\frac{\|(\operatorname{code} \mathrm{A} \otimes \operatorname{codeB}) \cap \operatorname{mask} \mathrm{A} \cap \operatorname{maskB}\|}{\|\operatorname{mask} \mathrm{A} \cap \operatorname{maskB}\|}$.

The denominator tallies the total number of phase bits that mattered in iris comparisons after artifacts such as eyelashes and specular reflections were discounted, so the resulting $\mathrm{HD}$ is a fractional measure of dissimilarity; 0 would represent a perfect match. The Boolean operators $\otimes$ and $\cap$ are applied in vector form to binary strings of length up to the word length of the CPU, as a single machine instruction. Thus, for example, on an ordinary 32-bit machine, any two integers between 0 and 4 billion can be XOR'ed in a single machine instruction to generate a third such integer, each of whose bits in a binary expansion is the XOR of the corresponding pair of bits of the original two integers. This implementation of Eq. (3) in parallel 32-bit chunks enables extremely rapid comparisons of iris codes when searching through a large database to find a match. On a $300 \mathrm{MHz}$ $\mathrm{CPU}$, such exhaustive searches are performed at a rate of about 100,000 irises/s.

Since any given bit in the phase code for an iris is equally likely to be 1 or 0 , and since different irises are uncorrelated, the expected proportion of agreeing bits between the codes for two different irises is $\mathrm{HD}=0.500$. The histogram in Fig. 4 shows the distribution of HDs obtained from 9.1 million comparisons between different pairings of iris images acquired by licensees of these algorithms in the UK, the USA, Japan, and Korea. There were 4258 different iris images, including 10 each of one subset of 70 eyes. Excluding those duplicates of $(700 \times 9)$ same-eye comparisons, and not double-counting pairs, and not comparing any image with itself, the total number of unique pairings between different eye images whose HDs could be computed was $((4258 \times 4257-700 \times 9) / 2)=9,060,003$. Their observed mean HD was $p=0.499$ with the standard deviation $\sigma=0.0317$; their full distribution in Fig. 4 corresponds to a fractional binomial having $N=p(1-p) / \sigma^{2}=249$ degrees-of-freedom, as shown by the solid curve. The extremely close fit of the theoretical fractional binomial to the observed distribution is a consequence of the fact that each comparison between two phase code bits from two different irises is essentially a Bernoulli trial, albeit with correlations between successive "coin tosses".

In the phase code for any given iris, only small subsets of bits are mutually independent due to the internal correlations, especially radial, within an iris. (If all $N=2048$ phase bits were independent, then the distribution in Fig. 4 would be very much sharper, with an expected standard deviation of only $\sqrt{p(1-p) / N}=0.011$ and so the HD interval between 0.49 and 0.51 would contain most of the distribution.) Bernoulli trials that are correlated (see Ref. [12]) remain binomially distributed but with a reduction in $N$, the effective number of tosses, and hence an increase in the $\sigma$ of the normalized HD distribution. The form and width of the HD distribution in Fig. 4 tell us that the amount of difference between the phase codes for different irises is distributed equivalently to runs of 249 tosses of a fair coin (Bernoulli trials with $p=0.5, N=249$ ). Expressing this variation as 


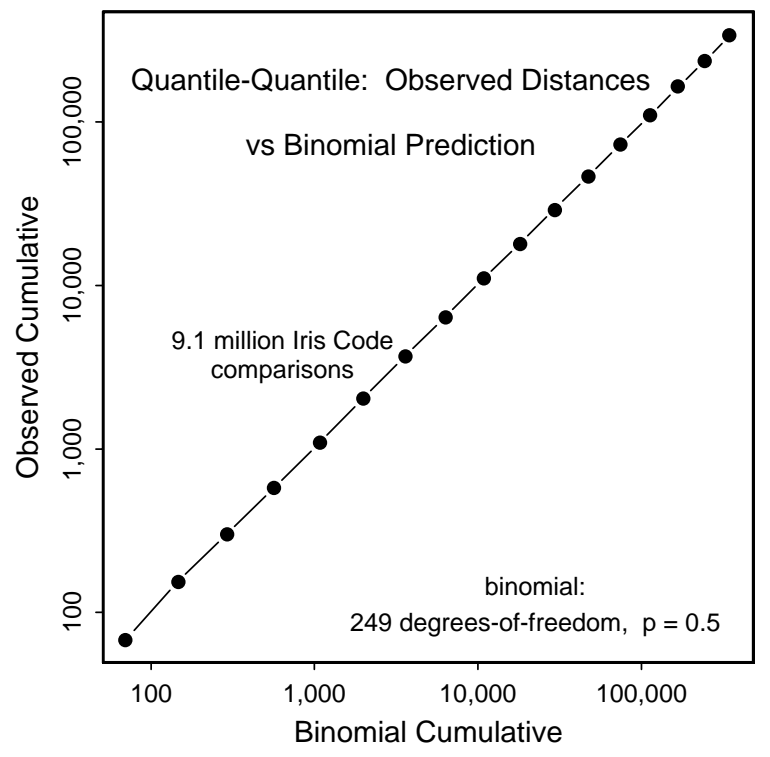

Fig. 5. Quantile-quantile plot of the observed cumulatives under the left tail of the histogram in Fig. 4, versus the predicted cumulatives under the theoretical binomial distribution. Their close agreement over several orders of magnitude strongly confirms the binomial model for phase bit comparisons between different irises.

a discrimination entropy (see Ref. [13]), and using typical iris and pupil diameters of 11 and $5 \mathrm{~mm}$, respectively, the observed amount of statistical variability among different iris patterns corresponds to an information density of about $3.2 \mathrm{bits} / \mathrm{mm}^{2}$ on the iris.

The theoretical binomial distribution plotted as the solid curve in Fig. 4 has the fractional functional form

$f(x)=\frac{N !}{m !(N-m) !} p^{m}(1-p)^{(N-m)}$,

where $N=249, p=0.5$, and $x=m / N$ is the outcome fraction of $N$ Bernoulli trials (e.g. coin tosses that are "heads" in each run). In our case, $x$ is the HD, the fraction of phase bits that happen to disagree when two different irises are compared. To validate such a statistical model we must also study the behaviour of the tails, by examining quantile-quantile plots of the observed cumulatives versus the theoretically predicted cumulatives from zero up to sequential points in the tail. Such a "Q-Q" plot is given in Fig. 5. The perfect linear relationship indicates precise agreement between model and data, over a range of almost four orders of magnitude. It is clear from both Figs. 4 and 5 that the extremely rapid attenuation of the tails of binomial distributions when $N$ is so large makes it extremely improbable that two different irises might disagree by chance in fewer than at least a third of their bits. Computing the cumulative of $f(x)$ from 0 to 0.333 indicates that the probability of such an event is about 1 in 16 million. (Of the 9.1 million iris comparisons plotted in the histogram of Fig. 4, the smallest HD observed was 0.334.) The binomial cumulative from 0 to just 0.300 is 1 in 10 bil- lion or roughly the planetary number of human eyes.Thus, even the observation of a relatively poor degree of match between the phase codes for two different iris images (say, $70 \%$ agreement or $\mathrm{HD}=0.300$ ) would still provide extraordinarily compelling evidence of identity, because this test of statistical independence is still failed so convincingly.

Genetically identical eyes were also compared in the same manner, in order to discover the degree to which their textural patterns were correlated and hence genetically determined. A convenient source of genetically identical irises are the right and left pair from any given person; such pairs have the same genetic relationship as the four irises of monozygotic twins, or indeed the prospective $2 N$ irises of $N$ clones. Although eye colour is of course strongly determined genetically, as is overall iris appearance, the detailed patterns of genetically identical irises appear to be as uncorrelated as they are among unrelated eyes. Using the same methods as described above, 648 right/left iris pairs from 324 persons were compared pairwise. Their mean HD was 0.497 with standard deviation 0.031, and their distribution (Fig. 6) was statistically indistinguishable from the distribution for unrelated eyes (Fig. 4).

A set of six pairwise comparisons among the eyes of actual monozygotic twins also yielded a result (mean $\mathrm{HD}=$ 0.507 ) expected for unrelated eyes. It appears that the phenotypic random patterns visible in the human iris are almost entirely epigenetic.

\section{Recognizing irises regardless of size, position, and orientation}

Robust representations for pattern recognition must be invariant under transformations in the size, position, and orientation of the patterns. For the case of iris recognition, this means that we must create a representation that is invariant to the optical size of the iris in the image (which depends upon both the distance to the eye, and the camera optical magnification factor); the size of the pupil within the iris (which introduces a non-affine pattern deformation); the location of the iris within the image; and the iris orientation, which depends upon head tilt, torsional eye rotation within its socket (cyclovergence), and camera angles, compounded with imaging through pan/tilt eye-finding mirrors that introduce additional image rotation factors as a function of eye position, camera position, and mirror angles. Fortunately, invariance to all of these factors can readily be achieved.

For on-axis but possibly rotated iris images, it is natural to use a projected pseudo-polar coordinate system. The polar coordinate grid is not necessarily concentric, since in most eyes the pupil is not central in the iris; it is not unusual for its nasal displacement to be as much as $15 \%$. This coordinate system can be described as doubly-dimensionless: the polar variable, angle, is inherently dimensionless, but in this case the radial variable is also dimensionless, because it varies from the pupillary boundary to the limbus always as a unit 
Genetically Identical Eyes Have Uncorrelated IrisCodes

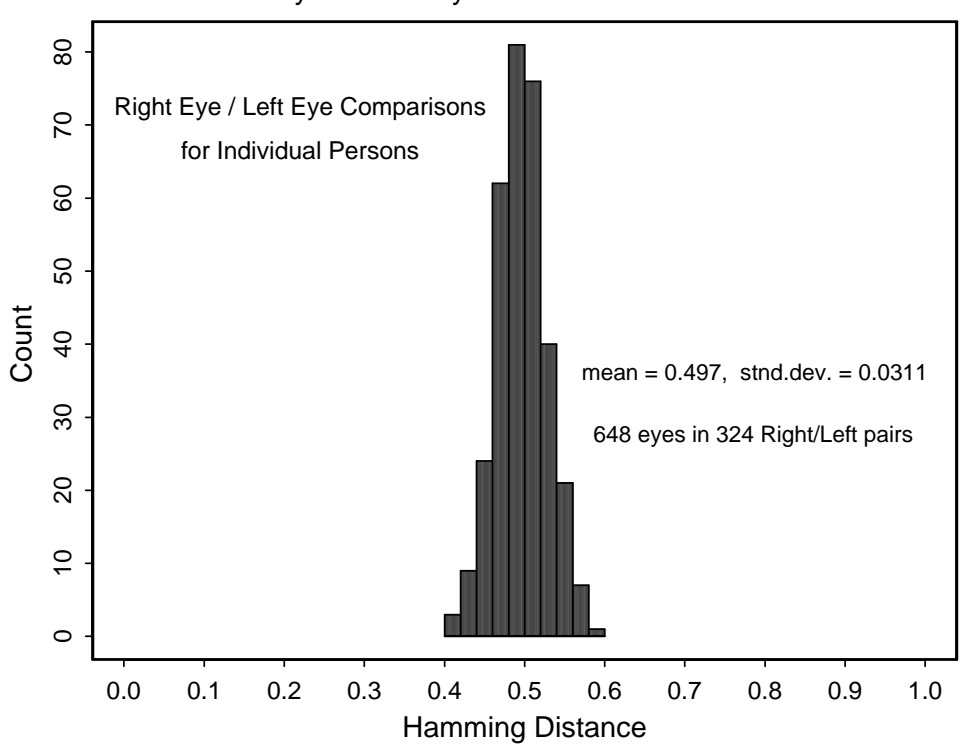

Fig. 6. Distribution of Hamming distances between genetically identical irises in 648 paired eyes from 324 persons. The data are statistically indistinguishable from that shown in Fig. 4 comparing unrelated irises. Unlike eye colour, the phase structure of iris patterns therefore appears to be epigenetic, arising from random events and circumstances in the morphogenesis of this tissue.

interval $[0,1]$. The dilation and constriction of the elastic meshwork of the iris when the pupil changes size is intrinsically modelled by this coordinate system as the stretching of a homogeneous rubber sheet, having the topology of an annulus anchored along its outer perimeter, with tension controlled by an (off-centred) interior ring of variable radius.

The homogeneous rubber sheet model assigns to each point on the iris, regardless of its size and pupillary dilation, a pair of real coordinates $(r, \theta)$ where $r$ lies on the unit interval $[0,1]$ and $\theta$ is angle $[0,2 \pi]$. The remapping of the iris image $I(x, y)$ from raw cartesian coordinates $(x, y)$ to the dimensionless non-concentric polar coordinate system $(r, \theta)$ can be represented as

$I(x(r, \theta), y(r, \theta)) \rightarrow I(r, \theta)$,

where $x(r, \theta)$ and $y(r, \theta)$ are defined as linear combinations of both the set of pupillary boundary points $\left(x_{p}(\theta), y_{p}(\theta)\right)$ and the set of limbus boundary points along the outer perimeter of the iris $\left(x_{s}(\theta), y_{s}(\theta)\right)$ bordering the sclera, both of which were detected by finding the maximum of the operator (1).

$x(r, \theta)=(1-r) x_{p}(\theta)+r x_{s}(\theta)$,

$y(r, \theta)=(1-r) y_{p}(\theta)+r y_{s}(\theta)$.

Since the radial coordinate ranges from the iris inner boundary to its outer boundary as a unit interval, it inherently corrects for the elastic pattern deformation in the iris when the pupil changes in size.
The localization of the iris and the coordinate system described above achieve invariance to the $2 \mathrm{D}$ position and size of the iris, and to the dilation of the pupil within the iris. However, it would not be invariant to the orientation of the iris within the image plane. The most efficient way to achieve iris recognition with orientation invariance is not to rotate the image itself using the Euler matrix, but rather to compute the iris phase code in a single canonical orientation and then to compare this very compact representation at many discrete orientations by cyclic scrolling of its angular variable. The statistical consequences of seeking the best match after numerous relative rotations of two iris codes are straightforward. Let $f_{0}(x)$ be the raw density distribution obtained for the HDs between different irises after comparing them only in a single relative orientation; for example, $f_{0}(x)$ might be the binomial defined in Eq. (4). Then $F_{0}(x)$, the cumulative of $f_{0}(x)$ from 0 to $x$, becomes the probability of getting a false match in such a test when using HD acceptance criterion $x$ :

$F_{0}(x)=\int_{0}^{x} f_{0}(x) \mathrm{d} x$

or, equivalently,

$f_{0}(x)=\frac{\mathrm{d}}{\mathrm{d} x} F_{0}(x)$.

Clearly, then, the probability of not making a false match when using criterion $x$ is $1-F_{0}(x)$ after a single test, and it is $\left[1-F_{0}(x)\right]^{n}$ after carrying out $n$ such tests independently at $n$ different relative orientations. It follows that the probability of a false match after a "best of $n$ " test of agreement, when 


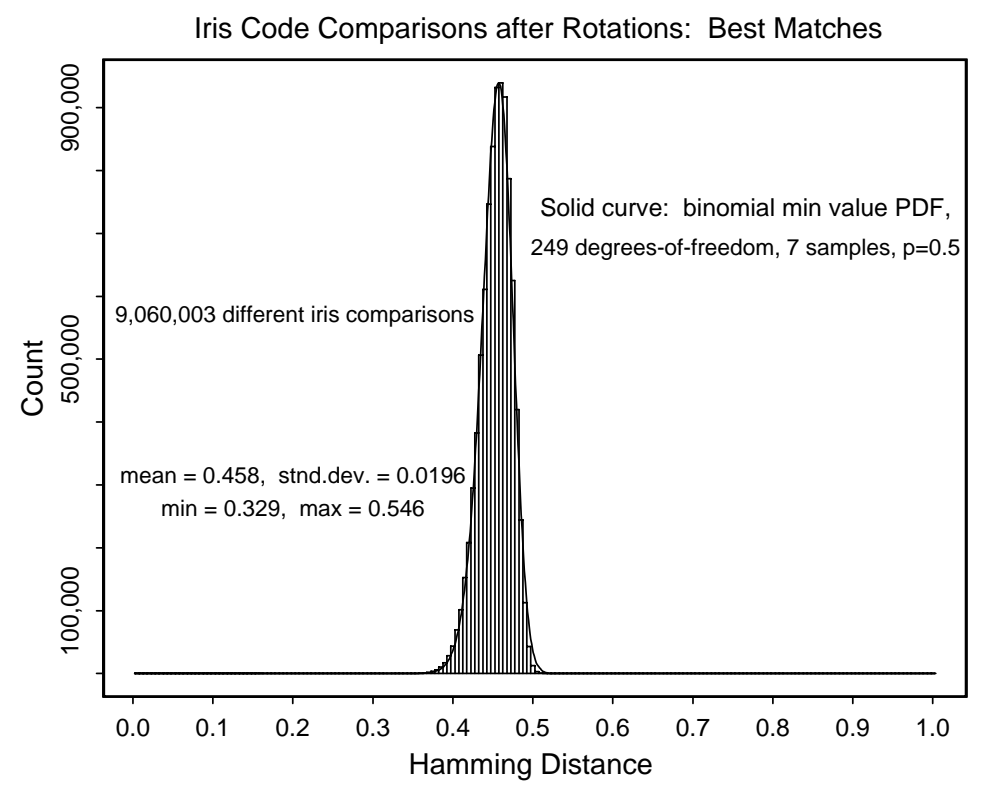

Fig. 7. Distribution of Hamming distances from the same set of 9.1 million comparisons as seen in Fig. 4, but allowing for seven relative rotations and preserving only the best match found for each pair. This "best of $n$ " test of agreement skews the distribution to the left and reduces its mean from about 0.5 to 0.458 . The solid curve is the theoretical prediction for such "extreme-value" sampling, as described by Eqs. (4) and (8)-(11).

using HD criterion $x$, regardless of the actual form of the raw unrotated distribution $f_{0}(x)$, is

$F_{n}(x)=1-\left[1-F_{0}(x)\right]^{n}$

and the expected density $f_{n}(x)$ associated with this cumulative is

$$
\begin{aligned}
f_{n}(x) & =\frac{\mathrm{d}}{\mathrm{d} x} F_{n}(x) \\
& =n f_{0}(x)\left[1-F_{0}(x)\right]^{n-1} .
\end{aligned}
$$

Each of the 9.1 million pairings of different iris images whose raw HD distribution was shown in Fig. 4, was submitted to further comparisons in each of the seven relative orientations. This generated 63 million HD outcomes, but in each group of seven associated with any one pair of irises, only the best match (smallest HD) was retained. The histogram of these new 9.1 million best HDs is shown in Fig. 7. Since only the smallest value in each group of seven samples was retained, the new distribution is skewed and biased to a lower mean value ( $\mathrm{HD}=0.458)$ as expected from the theory of extreme value sampling. The solid curve in Fig. 7 is a plot of Eq. (11), incorporating Eqs. (4) and (8) as its terms, and it shows an excellent fit between theory (binomial extreme value sampling) and data. The fact that the minimum HD observed among these millions of rotated comparisons was about 0.33 illustrates the extreme improbability that the phase sequences for two different irises might disagree in fewer than a third of their bits. This suggests that in order to identify people by their iris patterns with high confidence, we need to demand only a very forgiving degree of match (say, $\mathrm{HD} \leqslant 0.32$ ).

\section{Uniqueness of failing the test of statistical independence}

The statistical data and theory presented above show that we can perform iris recognition successfully just by a test of statistical independence. Any two different irises are statistically "guaranteed" to pass this test of independence, and any two images that fail this test (i.e. produce HD $\leqslant 0.32$ ) must be images of the same iris. Thus, it is the unique failure of the test of independence that is the basis for iris recognition.

It is informative to calculate the significance of any observed HD matching score, in terms of the likelihood that it could have arisen by chance from two different irises. These probabilities give a confidence level associated with any recognition decision. Fig. 8 shows the false match probabilities marked off in cumulatives along the tail of the distribution presented in Fig. 7 (same theoretical curve (11) as plotted in Fig. 7 and with the justification presented in Figs. 4 and 5). Table 1 enumerates the cumulatives of Eq. (11) (false match probabilities) as a more fine-grained function of HD decision criterion in the range between 0.26 and 0.40. Calculation of the large factorial terms in Eq. (4) was done with Stirling's approximation which errs by $<1 \%$ 
False Match Probabilities: Cumulatives under PDF

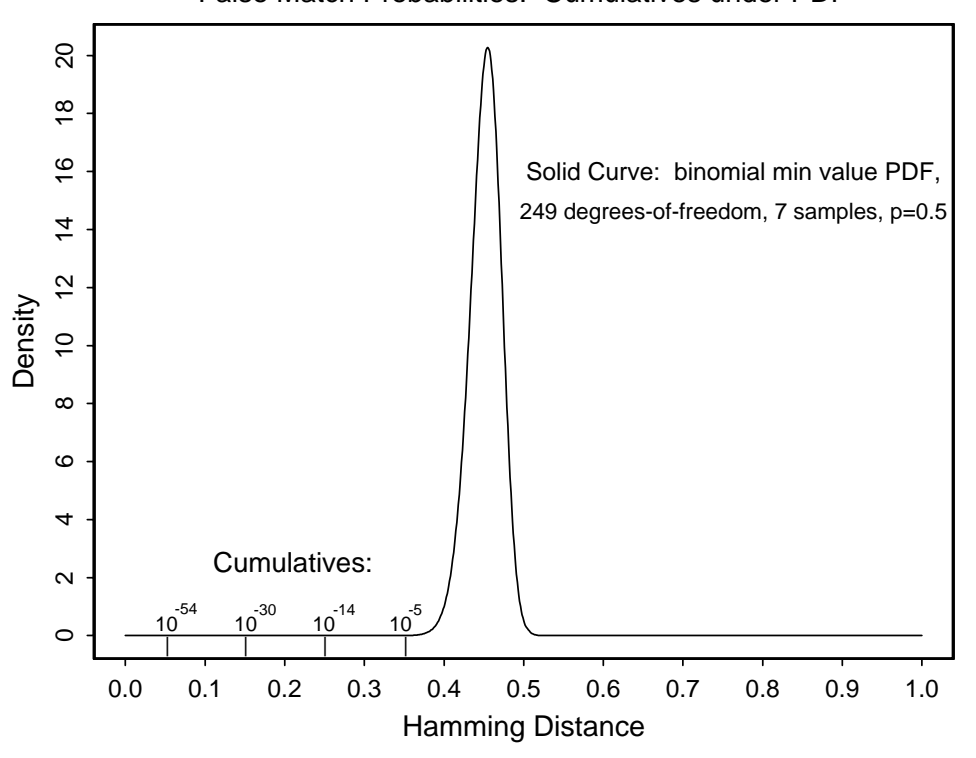

Fig. 8. Calculated cumulatives under the left tail of the distribution seen in Fig. 7, up to sequential points, using the same theoretical PDF described by Eqs. (4) and (8)-(11). The extremely rapid attenuation of these cumulatives reflects the binomial combinatorics with large $N$ in Eq. (4). This accounts for the astronomic confidence levels against a false match when persons are recognized by failing this test of statistical independence.

Table 1

Cumulatives under Eq. (11) giving false match probabilities for various HD criteria

\begin{tabular}{ll}
\hline HD criterion & Odds of false match \\
\hline 0.26 & 1 in $10^{13}$ \\
0.27 & 1 in $10^{12}$ \\
0.28 & 1 in $10^{11}$ \\
0.29 & 1 in 13 billion \\
0.30 & 1 in 1.5 billion \\
0.31 & 1 in 185 million \\
0.32 & 1 in 26 million \\
0.33 & 1 in 4 million \\
0.34 & 1 in 690,000 \\
0.35 & 1 in 133,000 \\
0.36 & 1 in 28,000 \\
0.37 & 1 in 6750 \\
0.38 & 1 in 1780 \\
0.39 & 1 in 520 \\
0.40 & 1 in 170 \\
\hline
\end{tabular}

for $n \geqslant 9$ :

$n ! \approx \exp \left(n \ln (n)-n+\frac{1}{2} \ln (2 \pi n)\right)$.

The practical importance of the astronomic odds against a false match when the match quality is better than about $\mathrm{HD} \leqslant 0.32$, as shown in Fig. 8 and in Table 1 , is that such high confidence levels allow very large databases to be searched exhaustively without succumbing to any of the many opportunities for suffering a false match. The requirements of operating in one-to-many "identification" mode are vastly more demanding than operating merely in one-to-one "verification" mode (in which an identity must first be explicitly asserted, which is then verified in a yes/no decision by comparison against just the single nominated template).

If $P_{1}$ is the false match probability for single one-to-one verification trials, then clearly $P_{N}$, the probability of making at least one false match when searching a database of $N$ unrelated patterns, is

$P_{N}=1-\left(1-P_{1}\right)^{N}$

because $\left(1-P_{1}\right)$ is the probability of not making a false match in single comparisons; this must happen $N$ independent times; and so $\left(1-P_{1}\right)^{N}$ is the probability that such a false match never occurs.

It is interesting to consider how a seemingly impressive one-to-one "verifier" would perform in exhaustive search mode once databases become larger than about 100 , in view of Eq. (13). For example, a face recognition algorithm that truly achieved $99.9 \%$ correct rejection when tested on non-identical faces, hence making only $0.1 \%$ false matches, would seem to be performing at a very impressive level because it must confuse no more than one-tenth of all identical twin pairs (since about $1 \%$ of all persons in the general population have an identical twin). But even with its $P_{1}=0.001$, how good would it be for searching large databases?

Using Eq. (13) we see that when the search database size has reached merely $N=200$ unrelated faces, the probability of at least one false match among them is already 
$18 \%$. When the search database is just $N=2000$ unrelated faces, the probability of at least one false match has reached $86 \%$. Clearly identification is vastly more demanding than one-to-one verification, and even for moderate database sizes, merely "good" verifiers are of no use as identifiers. Observing the approximation that $P_{N} \approx N P_{1}$ for small $P_{1} \ll 1 / N \ll 1$, when searching a database of size $N$, an identifier needs to be roughly $N$ times better than a verifier to achieve comparable odds against making false matches.

The algorithms for iris recognition exploit the extremely rapid attenuation of the HD distribution tail created by binomial combinatorics to accommodate very large database searches without suffering false matches. The decision threshold is adaptive, to maintain $P_{N}<10^{-6}$ regardless of how large the search database size $N$ is. As illustrated in Table 1, this means that if the search database contains 1 million different iris patterns, it is only necessary for the HD match criterion to adjust downwards from 0.32 to 0.27 in order to maintain still a net false match probability of $10^{-6}$ for this entire large database.

At the other extreme, it is remarkable that even if matches were accepted in which up to $40 \%$ of the bits disagreed, the false match rate would still be only about $0.5 \%$ and better than most biometrics (see Table 1 for $\mathrm{HD}=0.40$ ). This allows great tolerance for poor imaging conditions, such as may be unavoidable in covert use.

\section{Decision environment for iris recognition}

The overall "decidability" of the task of recognizing persons by their iris patterns is revealed by comparing the HD distributions for same versus for different irises. The left distribution in Fig. 9 shows the HDs computed between 7070 different pairs of same-eye images at different times, under different conditions, and usually with different cameras; and the right distribution shows the results of comparisons among different eyes. To the degree that one can confidently decide whether an observed sample belongs to the left or the right distribution in Fig. 9, iris recognition can be successfully performed. Such a dual distribution representation of the decision problem may be called the "decision environment", because it reveals the extent to which the two cases (same versus different) are separable and thus how reliably decisions can be made, since the overlap between the two distributions determines the error rates.

Whereas Fig. 9 shows the decision environment under less favourable conditions (images acquired by different camera platforms), Fig. 10 shows the decision environment under ideal (indeed, artificial) conditions. Subjects' eyes were imaged in a laboratory setting using always the same camera with fixed zoom factor and at fixed distance, and with fixed illumination. Not surprisingly, more than half of such image comparisons achieved an HD of 0.00 , and the average HD was a mere 0.019 . It is clear from the comparison of Figs. 9 and 10 that the "authentics" distribution for iris recog- nition (the similarity between different images of the same eye, as shown in the left-side distributions) depends very strongly upon the image acquisition conditions. However, the measured similarity for "imposters" (the right-side distribution) is apparently almost completely independent of imaging factors. Instead, it mainly reflects just the combinatorics of Bernoulli trials, as bits from independent binary sources (the phase codes for different irises) are compared.

For two-choice decision tasks (e.g. same versus different), the decidability index $d^{\prime}$ measures how well separated the two distributions are, since recognition errors would be caused by their overlap. If their two means are $\mu_{1}$ and $\mu_{2}$, and their two standard deviations are $\sigma_{1}$ and $\sigma_{2}$, then $d^{\prime}$ is defined as

$d^{\prime}=\frac{\left|\mu_{1}-\mu_{2}\right|}{\sqrt{\left(\sigma_{1}^{2}+\sigma_{2}^{2}\right) / 2}}$.

This measure of decidability is independent of how liberal or conservative is the acceptance threshold used. Rather, by measuring separation, it reflects the degree to which any improvement in (say) the false match error rate must be paid for by a worsening of the failure-to-match error rate. The measured decidability for iris recognition is $d^{\prime}=7.3$ for the non-ideal (crossed platform) conditions presented in Fig. 9, and it is $d^{\prime}=14.1$ for the ideal imaging conditions presented in Fig. 10.

Based on the left-side distributions in Figs. 9 and 10, one could also calculate a table of probabilities of failure to match [14], as a function of HD match criterion, just as we did earlier in Table 1 for false match probabilities based on the right-side distribution. However, such estimates may not be stable because the same-eye distributions (left-side) depend strongly on the quality of imaging (e.g. motion blur, focus, noise, etc.) and would be different for different optical platforms. As illustrated earlier by the badly defocused image of Fig. 3, phase bits are still set randomly with binomial statistics in poor imaging, and so the right-side distribution is the stable asymptotic form both in the case of well imaged irises (Fig. 10) and poorly imaged irises (Fig. 9). Imaging quality determines how much the same-iris distribution evolves and migrates leftward, away from the asymptotic different-iris distribution on the right. In any case, we note that for the 7070 same-iris comparisons shown in Fig. 9, their highest HD was 0.327 which is below the smallest HD observed in even the 9.1 million comparisons between different irises. Thus, a decision criterion slightly below 0.33 produces a correct match rate of $100 \%$ and no false matches for the empirical data sets shown. At a criterion of 0.33 , using the cumulatives of Eq. (11) as tabulated in Table 1, the theoretical false match probability is 1 in 4 million.

Notwithstanding this diversity among iris patterns and their apparent singularity because of so many dimensions of random variation, their utility as a basis for automatic personal identification would depend upon their relative stability over time. There is a popular belief that the iris changes systematically with one's health or personality, and 
Decision Environment for Iris Recognition: Non-Ideal Imaging

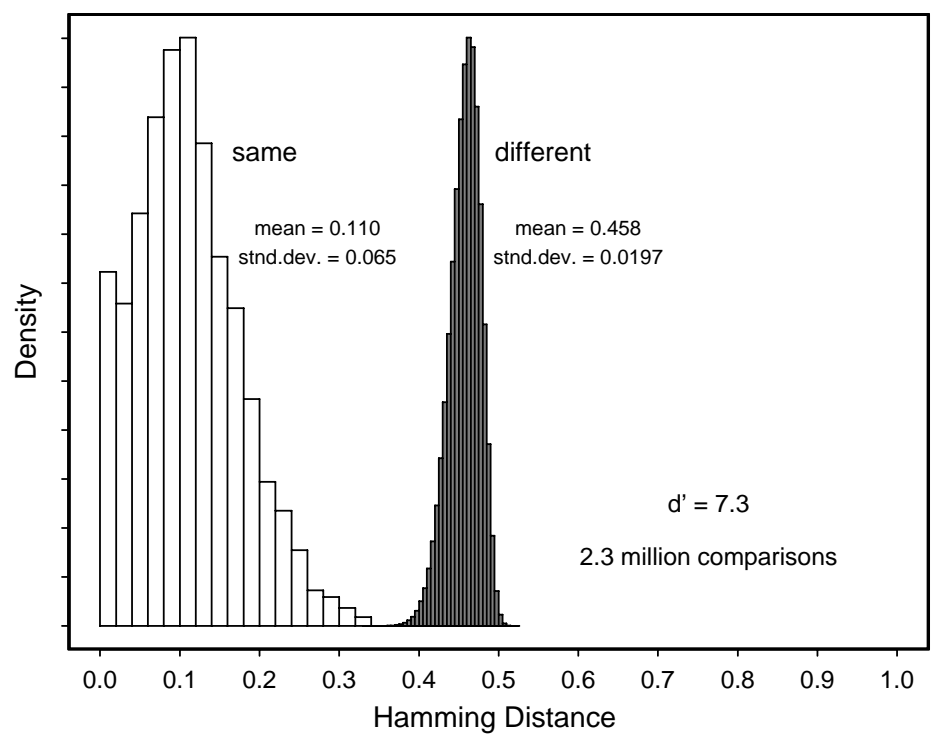

Fig. 9. The decision environment for iris recognition under relatively unfavourable conditions, using images acquired at different distances, and by different optical platforms.

Decision Environment for Iris Recognition: Ideal Imaging

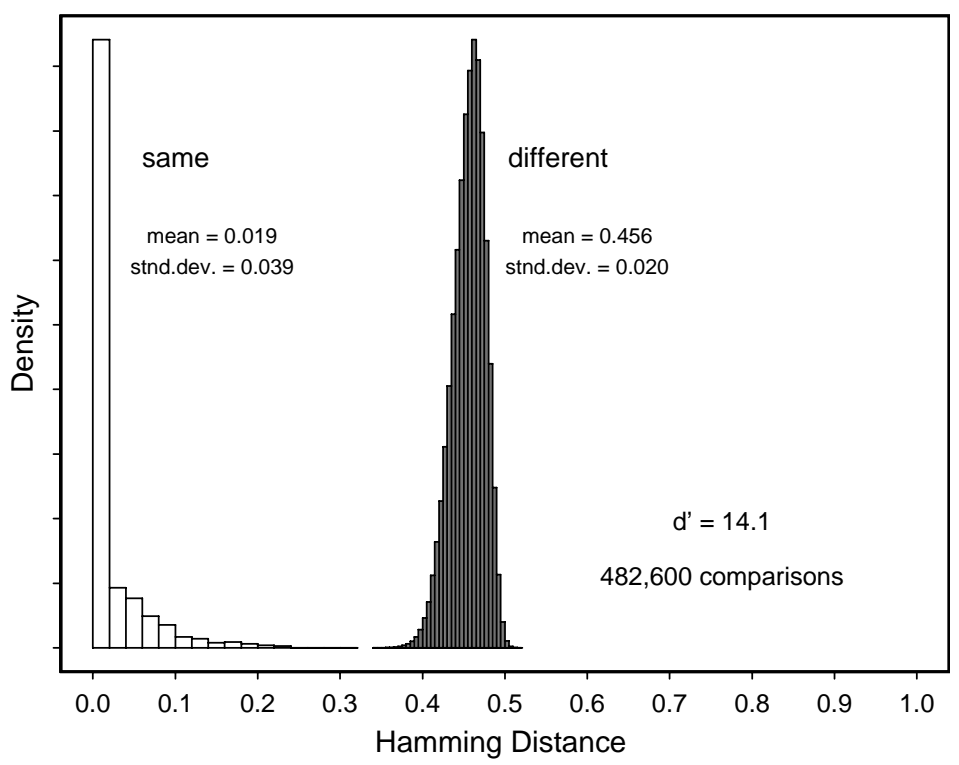

Fig. 10. The decision environment for iris recognition under very favourable conditions, using always the same camera, distance, and lighting, in a laboratory setting.

even that its detailed features reveal the states of individual organs ("iridology"); but such claims have been discredited (see e.g. Refs. $[15,16]$ ) as medical fraud. In any case, the recognition principle described here is intrinsically tolerant of a large proportion of the iris information being corrupted, say up to about a third, without significantly impairing the inference of personal identity by the simple test of statistical independence. 
Table 2

Speeds of various stages in the iris recognition process.

\begin{tabular}{ll}
\hline Operation & Execution time \\
\hline Assessing image focus & $15 \mathrm{~ms}$ \\
Scrubbing specular reflections & $56 \mathrm{~ms}$ \\
Localizing the eye and iris & $90 \mathrm{~ms}$ \\
Fitting the pupillary boundary & $12 \mathrm{~ms}$ \\
Detecting and fitting both the eyelids & $93 \mathrm{~ms}$ \\
Removing eyelashes and contact lens artifacts & $78 \mathrm{~ms}$ \\
Demodulation and iriscode creation & $102 \mathrm{~ms}$ \\
XOR comparison of any two iriscodes & $10 \mu \mathrm{s}$ \\
\hline
\end{tabular}

\section{Speed performance summary}

On a $300 \mathrm{MHz}$ Sun workstation, the execution times for the critical steps in iris recognition are as indicated in Table 2 , using optimized integer code.

The search engine can perform about 100,000 full comparisons between different irises per second, because of the efficient implementation of the matching process in terms of elementary Boolean operators $\otimes$ and $\cap$ acting in parallel on the computed phase bit sequences.

\section{Permutation of bytes to defeat replay attacks}

The test of statistical independence which is the basis of iris recognition is of course indifferent to the order in which the bytes of two iris codes are compared, provided that any permutation of byte order is identical for both iris codes. This is because each demodulating wavelet that sets a pair of phase bits is a point process, and no identifying information is embedded in the relationship between the bits computed from different wavelets. This "non-metric" property is not shared by other biometrics that are based on encoding some kind of spatial map. It creates a powerful means for preventing, or defeating, a "digital replay attack" in which an iris code transmitted electronically in a remote transaction might be captured by an eavesdropper and submitted subsequently as an impersonation, or replay, attack.

The 256 data bytes in an iris code are capable of 256 ! = $10^{507}$ different permutations of byte order. Provided that both the centrally enrolled iris code and the presenting one for any given transaction, any given application or as stored on any particular secured device, are permuted according to the same permutation table, then recognition can still be performed with results unaffected by the permutation. The massive number of $10^{507}$ possible cyclic permutations means that this process could even be performed on a daily, or hourly, basis without remotely exhausting the space of code permutations that can be generated by a congruential secret key. Moreover, all such concatenated permutations are simply just another permutation, so there is no need to store the long history of permutations; only the current resulting one. This scenario creates new security possibilities (e.g. an endless number of device-specific or application-specific iris codes generated from any given iris) for the manner in which iris recognition is deployed.

\section{Future developments}

The iris phase codes can be compressed without loss of information to about 50 bytes, and even to as little as 36 bytes by undersampling if one can tolerate a reduction in the number of degrees-of-freedom to about 102. However, there is little benefit to be gained from these information-theoretic manipulations to shorten the code, because of the bandwidth and memory storage capacities today of even portable devices and media.

The minimum resolution required for iris recognition remains to be explored. As implied by comparing the left-side distributions in Figs. 9 and 10, the main aspects of iris recognition that require further improvement are related to image acquisition. Since the iris is a small target, and a moving target, and one that finds bright illumination aversive, many compromises in imaging are required. For example, all optical parameters ( $F /$ number, focal depth of field, sensor SNR, necessary CCD integration time) are improved just by using more light, especially in the visible wavelengths. But for reasons mentioned earlier, this is not an option.

A further negative consequence of the need to use NIR illumination is that the sclera is often quite dark at these wavelengths (sometimes darker even than the iris) because the wall of the eye contains much blood, and hemoglobin has a strong absorption band around $830 \mathrm{~nm}$. The comparative darkness of the sclera in such wavelengths makes it more difficult to localize accurately the iris/sclera boundary. The desire to acquire eye images at distances of about a meter demands considerable magnification, but this also works against all of the optical parameters listed earlier. Yet despite these challenges, the huge tolerance for error in the encoded bit streams (up to about 33\%) is a basis for optimism. The power of the simple test of statistical independence reveals (as Oscar Wilde [17] might have said) the importance, and the benefits, of being random.

\section{References}

[1] A.N. Kolmogorov, Three approaches to the quantitative definition of information, Probl. Inform. Transm. 1 (1965) 4-7.

[2] P.N. Belhumer, J.P. Hespanha, D.J. Kriegman, Eigenfaces vs. fisherfaces: recognition using class-specific linear projection, IEEE Trans. Pattern Anal. Mach. Intell. 19 (7) (1977) 711720.

[3] Y. Adini, Y. Moses, S. Ullman, Face recognition: the problem of compensating for changes in illumination direction, IEEE Trans. Pattern Anal. Mach. Intell. 19 (7) (1997) 721-732. 
[4] P.J. Phillips, H. Moon, S.A. Rizvi, P.J. Rauss, The FERET evaluation methodology for face-recognition algorithms, IEEE Trans. Pattern Anal. Mach. Intell. 22 (10) (2000) 1090-1104.

[5] A. Pentland, T. Choudhury, Face recognition for smart environments, Computer 33 (2) (2000) 50-55.

[6] P. Kronfeld, Gross anatomy and embryology of the eye, in: H. Davson (Ed.), The Eye, Academic Press, London, 1962.

[7] J. Daugman, High confidence visual recognition of persons by a test of statistical independence, IEEE Trans. Pattern Anal. Mach. Intell. 15 (11) (1993) 1148-1161.

[8] J. Daugman, C. Downing, Demodulation, predictive coding, and spatial vision, J. Opt. Soc. Amer. A 12 (4) (1995) 641-660.

[9] J. Daugman, Two-dimensional spectral analysis of cortical receptive field profiles, Vision Res. 20 (10) (1980) 847-856.

[10] J. Daugman, Uncertainty relation for resolution in space, spatial frequency, and orientation optimized by two-dimensional visual cortical filters, J. Opt. Soc. Amer. A 2 (7) (1985) 1160-1169.
[11] J. Daugman, Complete discrete 2D Gabor transforms by neural networks for image analysis and compression, IEEE Trans. Acoustics, Speech, Signal Process. 36 (7) (1988) 1169-1179.

[12] R. Viveros, K. Balasubramanian, N. Balakrishnan, Binomial and negative binomial analogues under correlated Bernoulli trials, Amer. Statist. 48 (3) (1984) 243-247.

[13] T. Cover, J. Thomas, Elements of Information Theory, Wiley, New York, 1991.

[14] J. Daugman, U.S. Patent No. 5,291,560: Biometric Personal Identification System Based on Iris Analysis, 1 March 1994.

[15] L. Berggren, Iridology: a critical review, Acta Ophthalmol. 63 (1) (1985) 1-8.

[16] A. Simon, D.M. Worthen, J.A. Mitas, An evaluation of iridology, J. Amer. Med. Assoc. 242 (1979) 1385-1387.

[17] O. Wilde, The Importance of Being Ernest, Leonard Smithers, London, 1895. (Ernest): "Lady Bracknell, I hate to seem inquisitive, but would you kindly inform me who I am?"

\begin{abstract}
About the Author-JOHN DAUGMAN is a faculty member at Cambridge University, where he teaches courses in Information Theory, Computer Vision, Continuous Mathematics, and Neural Computing. He received his degrees at Harvard University, where he also subsequently taught on the faculty. He has served as an editor of several journals including IEEE Trans. Pattern Analysis and Machine Intelligence; Network Computation in Neural Systems; Cognitive Brain Research; and Journal of Computation and Mathematics. His academic awards include the US National Science Foundation's Presidential Young Investigator Award; the inaugural Toshiba Endowed Chair of the Tokyo Institute of Technology; and the Order of the British Empire from Her Majesty Queen Elizabeth II. Daugman is the inventor of iris recognition for personal identification, for which he received US Patent 5,291,560 in 1994. These algorithms won the 1997 Information Technology Award and Medal of the British Computer Society; the 2000 Technology Innovation Award of the US Smithsonian Museum; and the "Millenium Product" designation by the UK Design Council.
\end{abstract}

